\title{
DOUBLE SECTIONS, DOMINATING MAPS, AND THE JACOBIAN FIBRATION
}

\author{
By Gregery T. BuZzard and Steven S.-Y. Lu
}

\begin{abstract}
We give two parametrized versions of the uniformization theorem of a noncompact, nonhyperbolic Riemann surface using different but complementary methods. The first constructs the uniformizing maps directly in terms of coordinates via classical complex analysis and provides a canonical form for the double sections of a conic bundle over a noncompact complex curve. The second version, which is coordinate independent, works over any complex curve and is obtained by extending Kodaira's theory of the Jacobian fibration to a family of singular algebraic curves constructed via algebraic geometry. Then, using the results obtained with the Jacobian fibration, we give two equivalent conditions for a complex analytic surface nonhyperbolically fibered over a complex curve to be holomorphically dominable by $\mathbb{C}^{2}$ : We show that this dominability is equivalent to the apparently weaker condition of the existence of a Zariski dense image of $\mathbb{C}$ and equivalent to the quasiprojectivity of the base curve together with the nonnegativity of the orbifold Euler characteristic. We discuss also the sharpness of our result in various contexts as well as the lack of connection to the fundamental group.
\end{abstract}

1. Introduction. As is well known, there exist nonconstant holomorphic maps from the plane into the complex sphere $\mathbb{P}^{1}$ minus two points, the simplest example of which is an explicit realization of the uniformization map given by applying the exponential map and then composing with a Mobius transformation taking 0 and $\infty$ to the two given punctures. Likewise, we can map the plane into the sphere minus one point by simply applying directly a Mobius transformation taking $\infty$ to this puncture.

In this paper we prove two parametrized versions of this uniformization result using different methods. We first present a slightly easier version using complex analysis, which allows us to construct the uniformizing maps more or less explicitly in terms of the initial coordinates given. Then we give the more general and coordinate invariant version, which we prove by extending Kodaira's theory of the Jacobian fibration to a family of singular curves constructed via algebraic geometry.

Finally, using the results obtained with the Jacobian fibration, we obtain two equivalent conditions for a complex surface nonhyperbolically fibered over a curve to be holomorphically dominable by $\mathbb{C}^{2}$. In Theorem 1.7 we show that this dominability is equivalent to the apparently weaker condition of the existence of

Manuscript received October 18, 1999; revised December 22, 1999.

Research of the first author supported in part by an NSF grant. Research of the second author supported in part by an NSERC grant.

American Journal of Mathematics 122 (2000), 1061-1084. 
a Zariski dense image of $\mathbb{C}$ and equivalent to the quasiprojectivity of the base curve together with the nonnegativity of the orbifold Euler characteristic.

We start with the following definition.

Definition 1.1. Let $R$ be a Riemann surface and let $D \subset R \times \mathbb{P}^{1}$ be a 1dimensional complex subvariety such that for generic $z \in R$ the fiber $D_{z}=$ $D \cap\left(\{z\} \times \mathbb{P}^{1}\right)$ has two points. Then $D$ is called a double section over $R$.

Our first parametrized version of uniformization is that, given a double section $D$ over a noncompact Riemann surface $R$, there is a fiber preserving holomorphic map from $R \times \mathbb{C}$ to the complement of $D$ which on each fiber is a uniformizing map as described above.

THEOREM 1.2. Let D be a double section over a noncompact Riemann surface $R$. Then there exists a holomorphic map $F: R \times \mathbb{C} \rightarrow\left(R \times \mathbb{P}^{1}\right) \backslash D$ of the form $F(z, w)=(z, H(z, w))$. Moreover, if $\# D_{z}=2$, then $H(z, w)=M_{z}\left(\exp \left(c_{z} w\right)\right)$, where $M_{z}$ is a Mobius transformation mapping $O$ and $\infty$ to the two points in $D_{z}$ and $c_{z}$ is a nonzero constant depending on $z$, while if $\# D_{z}=1$, then $H(z, w)=M_{z}(w)$ where $M_{z}$ is a Mobius transformation mapping $\infty$ to the point in $D_{z}$.

We will obtain this theorem by first proving a weaker result.

THEOREM 1.3. Let $D$ be a double section over a noncompact Riemann surface $R$. Then there exists a holomorphic function $\sigma: R \rightarrow \mathbb{P}^{1}$ whose graph, a holomorphic section over $R$, is disjoint from $D$.

As will be shown in Section 5, if we replace $D$ by a triple section, then this theorem is no longer true.

Note that any double section $D$ necessarily has the form

$$
D=\left\{(z, w): a(z) w^{2}+b(z) w+c(z)=0\right\}
$$

for holomorphic $a, b, c$. But, as a corollary of Theorem 1.3, we can always put $D$ into a normal form in the case $R$ is noncompact:

COROLlARY 1.4. Let D be a double section over a noncompact Riemann surface $R$. Then after a biholomorphic change of variables on $R \times \mathbb{P}^{1}$ preserving each fiber, there is a holomorphic function $h$ on $R$ such that $D$ has the form

$$
D=\left\{(z, w): w^{2}=h(z)\right\}
$$

We now give the more general version of our simultaneous uniformization result. Let $p: P \rightarrow R$ define a $\mathbb{P}^{1}$ bundle over a Riemann surface $R$. We may define a double section $D$ of $p$ as before. However, we will still reserve the term "double section over $R$ " to mean that $p$ defines a trivial holomorphic $\mathbb{P}^{1}$ bundle with an explicit trivialization, which can always be realized when $R$ is 
noncompact. Associated with $(P, D)$ is a Jacobian fibration, to be introduced in Section 5, which is a complex analytic surface obtained as a holomorphic quotient of a line bundle over $R$. The following theorem states that if $p$ has a section avoiding $D$, which as seen above is true when $R$ is noncompact, then $P \backslash D$ is isomorphic to this Jacobian fibration.

THEOREM 1.5. Let $p: P \rightarrow R$ define a holomorphic $\mathbb{P}^{1}$ bundle over a Riemann surface $R$ with a double section $D$. If $p$ has a section $\sigma$ that avoids $D$ (which is necessarily the case when $R$ is noncompact), then there is a holomorphic line bundle $L$ over $R$ and a holomorphic map $F: L \rightarrow P \backslash D$ preserving each fiber such that $F_{z}: L_{z} \rightarrow \mathbb{P}^{1} \backslash D_{z}$ is isomorphic to the universal covering map for all $z \in R$. Moreover, $F$ and $L$ are canonically determined by $(p, D, \sigma)$ and $F$ maps the zero-section of $L$ to $\sigma$.

Readers interested in the more abstract methods of algebraic geometry and the precise setting as detailed in the proof of this theorem should see Section 3. However, Section 3 can be skipped without losing continuity. This version implies the previous theorems by using this theorem in a local way, then solving an additive Cousin problem to obtain a global section as in Theorem 1.3. This is done in Section 3 as a corollary to Proposition 3.3.

The Jacobian fibration to be introduced in Section 3 is analogous to and indeed based on the one introduced by Kodaira [Kod, Kod1, Kod2] in his study of elliptic fibrations. This work of Kodaira served as an important foundation for the modern classification theory of compact complex surfaces. The Jacobian fibration introduced in the current paper is intended to serve as a precursor to a wider theory in which noncompact compactifiable complex manifolds may be studied more effectively and in greater detail and to provide motivation for studying the Jacobian fibration in the realm of families of singular varieties.

The motivation for this paper came from ideas contained in [BL]. In that paper we showed that for many algebraic surfaces, the existence of a Zariski dense image of $\mathbb{C}$ is equivalent to the existence of a dominating map from $\mathbb{C}^{2}$ into the surface (a dominating map is a holomorphic map with full rank generically). However, in that paper we did not resolve completely the question of the existence of a Zariski dense image of $\mathbb{C}$ in the complement of a general double section. The theorems above resolve this problem completely.

Additionally, in keeping with the general strategy of [BL] to deal with the dominability question for arbitrary complex surfaces, we obtain the following result. It states roughly that given any discrete set of jet prescriptions of sections over $R$, we can construct a dominating map, $F$, whose image avoids the double section outside the given discrete set while at the same time realizing the jet prescriptions in the following sense: if $c$ is a point in $R$ with a corresponding jet prescription and $\sigma$ is a holomorphic function in a neighborhood of $c$, then $F(z, \sigma(z))$ has the given jet prescription at $z=c$. 
THEOREM 1.6. Let $D$ be a double section over a noncompact Riemann surface $R$. Let $E$ be a discrete subset of $R$, and for each $c \in E$, let $P_{c}(z)$ be a Laurent polynomial in powers of $z-c$. Then there exists a holomorphic map $F$ from $R \times \mathbb{C}$ into $R \times \mathbb{P}^{1}$ of the form $F(z, w)=(z, H(z, w))$ such that if $c \in E$ and $\sigma$ is a holomorphic function in a neighborhood of $c$, then the Laurent expansion of $F(z, \sigma(z))$ agrees with $P_{c}(z)$ up to order $\operatorname{deg}\left(P_{c}\right)$, while if $c \notin E$, then $F(c, \cdot)$ maps $\mathbb{C}$ onto $\mathbb{P}^{1} \backslash D_{c}$ as in Theorem 1.2.

Coupled with results in [BL] and some geometry in Section 4.1 on orbifold Riemann surfaces, this allows us to give a complete geometric classification of nonhyperbolically fibered complex surfaces which are dominable by $\mathbb{C}^{2}$, i.e., which admit holomorphic maps from $\mathbb{C}^{2}$ having full rank somewhere. To state it, recall that a fibered surface $X$ is given by a surjective holomorphic map $f$ from the complex surface $X$ to a complex curve $C$ that extends to a proper holomorphic map on a complex surface $\bar{X}$ containing $X$ as a Zariski open subset. This gives a set $M_{s}$ of multiplicities for each point $s$ of $C$, comprising the various degrees of vanishing of $f$ (or multiplicities of $f$ ) at points of $X_{s}=f^{-1}(s)$ when $s$ is given as the origin in a local coordinate on $C$. Recall also that the classical multiplicity $n_{s}$ of the fiber $X_{s}$ is given as $\operatorname{gcd} M_{s}$. We define the new multiplicity $m_{s}$ at $s$ to be $\min M_{s}$. Then our result says that if the general fiber of $f$ is nonhyperbolic, then $X$ is dominable by $\mathbb{C}^{2}$ if and only if $C$ together with the $\mathbb{Q}$-divisor $A=\sum\left(1-1 / m_{s}\right) s$ is not hyperbolic as a marked Riemann surface. The latter happens precisely when $C$ is quasiprojective (with smooth compactification $\bar{C})$ and $\chi(C, A) \geq 0$, where the orbifold Euler characteristic is given by

$$
\chi(C, A):=2-2 g(\bar{C})-\#(\bar{C} \backslash C)-\operatorname{deg} A=e(C)-\operatorname{deg} A
$$

as in [BL].

THEOREM 1.7. Let $f: X \rightarrow C$ be a surjective holomorphic map defining $X$ into a fibered surface over the curve $C$. This imposes a branched orbifold structure on $C$ specified by a $\mathbb{Q}$-divisor $A=\sum\left(1-1 / m_{s}\right)$ s, where $m_{s}$ is the minimum multiplicity off at points above s, for each point $s \in C$. Assume that the general fiber $X_{c}$ is not hyperbolic, or equivalently, that $e\left(X_{c}\right) \geq 0$. Then the following are equivalent.

(a) $X$ is dominable by $\mathbb{C}^{2}$.

(b) $C$ is quasiprojective and $\chi(C, A) \geq 0$.

(c) There exists a holomorphic map of $\mathbb{C}$ to $X$ whose image is Zariski dense.

This paper is organized as follows. Section 2 proves Theorem 1.2 and Theorem 1.6 by first establishing Theorem 1.3 in Section 2.1. From this, Theorem 1.2, Theorem 1.6 and Corollary 1.4 follow naturally, as seen in Sections 2.2 and 2.3. The methods presented in Section 2 are completely concrete and allow us 
to write the dominating maps explicitly in terms of the initial coordinates. Section 3 presents the more abstract setting of Jacobian fibrations associated with our problem and uses this naturally coordinate invariant setting to prove Theorem 1.5. Section 4 recalls the setting for Theorem 1.7 from [BL], establishes a generalized orbifold version of Ahlfors' lemma, and uses Theorem 1.5 and the $n$ th-root fibration to establish Theorem 1.7. Finally, Section 5 provides some further remarks, including why our result is sharp to some extent, and gives further directions for investigation.

Acknowledgments. We are grateful to Bernard Shiffman for his aid in establishing this fruitful collaboration, and in particular for posing the problem that started it.

2. Dominating the complement of a double section. Throughout this section, $R$ will be assumed to be a noncompact Riemann surface, thus allowing us to use basic interpolation theorems on $R$ to construct the necessary maps.

2.1. A holomorphic section. In this section we prove Theorem 1.3. Let $D$ be a double section in $R \times \mathbb{P}^{1}$. Since there are only countably many $z \in R$ for which $\# D_{z}=1$, we can apply a map of the form $(z, w) \mapsto(z, M(w))$ for some Mobius transformation $M$ to ensure that if $\# D_{z}=1$, then $\infty \notin D_{z}$.

Let $E_{\infty}$ be the set of $z \in R$ such that $\infty \in D_{z}$. Then $E_{\infty}$ is discrete, so we may apply a map of the form $(z, w) \mapsto(z, w-c)$, where $c$ is a constant such that if $z \in E_{\infty}$ and $w \in D_{z}$, then $w-c \neq 0$. Hence we may assume that $D$ has been normalized so that if $z_{0} \in E_{\infty}$, then there is a neighborhood of $z_{0}$ such that in this neighborhood, $D$ is the union of the graphs of two functions of the form $u_{0}(z)$ and $u_{1}(z) /\left(z-z_{0}\right)^{m}$, where $u_{j}\left(z_{0}\right) \neq 0$ and $m \geq 1$.

Next, by [F, Theorems 26.3, 26.5], the classical theorems of Weierstrass and Mittag-Leffler are valid on $R$, so there exists $f_{1}$ holomorphic on $R$ such that if $z_{0} \in E_{\infty}$ with $u_{0}, u_{1}$ as described above, then $f_{1}(z)=\left(z-z_{0}\right)^{m}+O\left(\left|z-z_{0}\right|^{m+1}\right)$. Let $F_{1}(z, w)=\left(z, f_{1}(z) w\right)$, and let $C=F_{1}(D)$. Then $C$ is a double section and is contained in $R \times \mathbb{C}$. Moreover, if $z_{0} \in E_{\infty}$, then $C$ is locally the union of the graphs of $v_{0}(z)=u_{0}(z)\left(z-z_{0}\right)^{m}$ and $v_{1}(z)=u_{1}(z)$. Hence, suppose that $\tau$ is a meromorphic function on $R$ such that $\tau(z) \cap C_{z}=\emptyset$ if $z \notin E_{\infty}$, while if $z_{0} \in E_{\infty}$, then $\tau(z)=O\left(\left|z-z_{0}\right|^{m+1}\right)$. Then $F_{1}^{-1}(z, \tau(z))=\left(z, \tau(z) / f_{1}(z)\right)$ is a holomorphic section which avoids $D$ when $z \notin E_{\infty}$ and which equals $0 \in \mathbb{C} \backslash\left\{u_{0}(z)\right\}$ when $z \in E_{\infty}$. Thus $\sigma(z)=\tau(z) / f_{1}(z)$ gives a holomorphic section in the complement of $D$, so it suffices to construct $\tau$.

As a final simplification to $D$, we note that any symmetric polynomial in $v_{0}(z)$ and $v_{1}(z)$ gives a holomorphic function on $R$. So we may define the biholomorphic map $F_{2}(z, w)=\left(z, w-\left(v_{0}(z)+v_{1}(z)\right) / 2\right)$ and let $B=F_{2}(C)$. Then $B$ has the form $B=\left\{(z, w): w^{2}=h(z)\right\}$, where $h(z)=\left(\left(v_{0}(z)-v_{1}(z)\right) / 2\right)^{2}=\left(\left(v_{0}(z)+\right.\right.$ 
$\left.\left.v_{1}(z)\right) / 2\right)^{2}-v_{0}(z) v_{1}(z)$ is a holomorphic function on $R$. Note that if $z_{0} \in E_{\infty}$, then

$$
F_{2}(z, \tau(z))=\left(z,-\frac{u_{0}(z)\left(z-z_{0}\right)^{m}+u_{1}(z)}{2}+O\left(\left|z-z_{0}\right|^{m+1}\right)\right.
$$

So it suffices to construct a meromorphic function, $\mu$, whose graph avoids $B$ except at points $z_{0} \in E_{\infty}$, at which points $\mu$ should have a development as in the second coordinate of (2.1).

To obtain the function $\mu$, we will first define a holomorphic function on $B$, which we will write in the form $g(z, \sqrt{h(z)})$. We then define

$$
\mu(z)=\sqrt{h(z)} \frac{g(z, \sqrt{h(z)})+g(z,-\sqrt{h(z)})}{g(z, \sqrt{h(z)})-g(z,-\sqrt{h(z)})} .
$$

Note that for each $z, \mu$ is independent of the choice of $\pm \sqrt{h(z)}$, so $\mu$ is a welldefined meromorphic function on $R$ (by the Big Picard's theorem for example). We will construct $g$ to ensure that $\mu$ avoids $B$ except at points in $E_{\infty}$, where $\mu$ has the form dictated by (2.1).

To this end, let $E_{0}=\left\{z: \# D_{z}=1\right\}=\left\{z: 0 \in B_{z}\right\}=\{z: h(z)=0\}$. We make the following requirements for the holomorphic function $g$ on $B$ :

(a) If $z_{0} \in E_{0}$, then $g(z, \sqrt{h(z)})=1+\sqrt{h(z)}+O(\sqrt{|z h(z)|})$.

(b) If $z_{0} \in E_{\infty}$, then taking $\sqrt{h(z)}=\left(v_{0}(z)-v_{1}(z)\right) / 2$, we have $g(z, \sqrt{h(z)})=$ $1+z^{m}+O\left(\left|z-z_{0}\right|^{m+1}\right)$ and $g(z,-\sqrt{h(z)})=a z^{m}+O\left(\left|z-z_{0}\right|^{m+1}\right)$, where $a=$ $u_{0}\left(z_{0}\right) / u_{1}\left(z_{0}\right)$.

(c) If $z_{0} \notin\left(E_{0} \cup E_{\infty}\right)$, then $g(z, \pm \sqrt{h(z)}) \neq 0$.

To obtain such a function $g$, let $\Psi: X \rightarrow B$ be the normalization of $B$, where $X$ is a noncompact Riemann surface (see e.g. [C]). By [F, Theorems 26.3, 26.5], the classical theorems of Weierstrass and Mittag-Leffler are valid on $X$. Hence given finite holomorphic jets at a discrete set of points in $X$, there is a function holomorphic on $X$ agreeing with the given jets and having no other zeros. This can be done, for instance, by first finding a Weierstrass function, $f$, having the prescribed zeros, dividing all of the jet data by the jets of $f$ at the appropriate points, then taking $\log$ of these new jets and interpolating to get a function $r$ having these $\log$ jets. Then $f \exp (r)$ has the desired jets and no other zeros. Since $\Psi$ is locally biholomorphic except where $h=0$, the data in parts (b) and (c) transfer directly via $\Psi$ to data on $X$. Hence we need focus only on part (a).

Suppose $z_{0} \in E_{0}$. Without loss we may take $z_{0}=0$. Suppose that $h(z)=$ $z^{m} h_{1}(z)$ with $m$ odd and $h_{1}(0) \neq 0$. Then after a local change of coordinates in $X$ and a choice of $\sqrt{h_{1}(0)}, \Psi$ has the form $\Psi(x)=\left(x^{2}, x^{m} \sqrt{h_{1}\left(x^{2}\right)}\right)$; i.e., $x=\sqrt{z}$. In this coordinate system, $\Psi^{-1}(z, \pm \sqrt{h(z)})= \pm x= \pm \sqrt{z}$, and we may take $\sqrt{h(z)}=x^{m} \sqrt{h_{1}\left(x^{2}\right)}$. Hence, if $g_{1}$ is holomorphic in a neighborhood of 0 
in $X$ and satisfies $g_{1}(x)=1+x^{m} \sqrt{h_{1}(0)}+O\left(|x|^{m+1}\right)$, then $g=g_{1} \circ \Psi^{-1}$ has the development given in (a) in a deleted neighborhood of 0 .

Suppose next that $h(z)=z^{2 m} h_{1}(z)$ with $h(0) \neq 0$. In this case, $B$ consists of two distinct components which intersect at the origin, and hence the origin lifts to two distinct points $x_{0}, x_{1}$ in $X$. Making a choice of $\sqrt{h_{1}(0)}$, we see that after a local change of coordinates near $x_{j}, \Psi$ has the form

$$
\Psi(x)=\left(x-x_{j},(-1)^{j}\left(x-x_{j}\right)^{m} \sqrt{h_{1}\left(x-x_{j}\right)}\right) .
$$

Hence $\Psi^{-1}\left(z,(-1)^{j} z^{m} \sqrt{h_{1}(z)}\right)=z+x_{j}$. As before, in $X$, if $g_{1}$ is holomorphic in a neighborhood of $x_{j}$ and satisfies $g_{1}(x)=1+(-1)^{j}\left(x-x_{j}\right)^{m} \sqrt{h_{1}(0)}+O\left(\left|x-x_{j}\right|^{m+1}\right)$, then $g=g_{1} \circ \Psi^{-1}$ satisfies the development in (a).

Hence the requirement in part (a) can be satisfied by specifying a discrete set of finite jets in $X$. As mentioned above, there exists a function $g_{1}$ holomorphic on $X$ agreeing with the given finite jets and having no other zeros. Then $g=g_{1} \circ \Psi^{-1}$ satisfies (b) and (c) and satisfies (a) in a deleted neighborhood of each $z_{0} \in E_{0}$.

Observe that for $z$ outside $E_{0} \cup E_{\infty}, \mu(z) \neq \pm \sqrt{h(z)}$ since $g(z, \pm \sqrt{h(z)}) \neq 0$ for either choice of \pm there. Hence to finish the proof we need show only that $\mu$ is meromorphic and nonzero at each $z_{0} \in E_{0}$, and that $\mu$ has the development given by (2.1) at points $z_{0} \in E_{\infty}$.

Suppose first that $z_{0} \in E_{0}$. As before, we may assume $z_{0}=0$ and that $h(z)=z^{m} h_{1}(z)$ with $h_{1}(0) \neq 0$ and $m \geq 1$. Recalling that $\mu$ is independent of the choice of $\pm \sqrt{h(z)}$, we have

$$
\begin{aligned}
\mu(z) & =\sqrt{h} \frac{(1+\sqrt{h})+(1-\sqrt{h})+O\left(|z|^{(m+1) / 2}\right)}{(1+\sqrt{h})-(1-\sqrt{h})+O\left(|z|^{(m+1) / 2}\right)} \\
& =1+O\left(|z|^{1 / 2}\right) .
\end{aligned}
$$

Hence $\mu$ has a removable singularity at $z_{0}=0$ and is nonzero there.

Finally, suppose that $z_{0} \in E_{\infty}$, and recall that in this case, $h(z) \neq 0$, and $\sqrt{h(z)}=\left(v_{0}(z)-v_{1}(z)\right) / 2$, where, assuming that $z_{0}=0$, we have $v_{0}(z)=z^{m} u_{0}(z)$ and $v_{1}(z)=u_{1}(z), u_{j}(0) \neq 0$, and the development in part (b) holds. Then

$$
\begin{aligned}
\mu(z) & =\left(\frac{v_{0}-v_{1}}{2}\right) \frac{1+(1+a) z^{m}+O\left(|z|^{m+1}\right)}{1+(1-a) z^{m}+O\left(|z|^{m+1}\right)} \\
& =\left(\frac{u_{0} z^{m}-u_{1}}{2}\right)\left(1+2 a z^{m}+O\left(|z|^{m+1}\right)\right) \\
& =-\frac{u_{0} z^{m}+u_{1}}{2}+u_{0} z^{m}-a u_{1} z^{m}+O\left(|z|^{m+1}\right) .
\end{aligned}
$$

Since $a=u_{0}\left(z_{0}\right) / u_{1}\left(z_{0}\right)$, we see that this last expression has the same form as 
the second coordinate in (2.1). So $\mu$ has all the desired properties, and hence applying $F_{2}^{-1}$ and $F_{1}^{-1}$ gives a holomorphic section avoiding $D$, as claimed.

2.2. Normal form and dominating map. In this section we prove Corollary 1.4 and use this to prove Theorem 1.2. The proof of Corollary 1.4 is essentially a matter of changing coordinates in $R \times \mathbb{P}^{1}$ so that the section provided by Theorem 1.3 becomes the infinity section in the new coordinates, then averaging to put the resulting section in the desired form. We provide the proof for completeness.

Proof of Corollary 1.4. Let $D$ be a double section over $R$. We first apply a map which is biholomorphic on $R \times \mathbb{P}^{1}$ and which maps $D$ into $R \times \mathbb{C}$. To construct such a map, note that by Theorem 1.3, there exists a function $\sigma$ meromorphic on $R$ such that the graph, $\Sigma$, of $\sigma$ in $R \times \mathbb{P}^{1}$ is disjoint from $D$. If $\sigma$ is holomorphic, we apply the map $M(z, w)=(z, 1 /(w-\sigma(z)))$, which is biholomorphic on $R \times \mathbb{P}^{1}$ and which takes $\Sigma$ to the infinity section and hence satisfies $M(D) \subset R \times \mathbb{C}$.

Otherwise, $\sigma$ is meromorphic but not holomorphic. Choose a function $\psi$ holomorphic on $R$ such that $\psi$ is zero precisely when $\sigma$ has either a zero or a pole, with the zeros of $\psi$ chosen so that $1 / \psi$ has the same principal part as $1 / \sigma$ if $\sigma$ has a 0 and $1 / \psi$ has the same principal part as $\sigma$ if $\sigma$ has a pole. Let $\phi(z)=(-1 / \sigma(z))+(1 / \psi(z))$, and let

$$
M(z, w)=\frac{\phi(z) w+1}{w-\sigma(z)}
$$

If $\sigma\left(z_{0}\right) \in \mathbb{C}$, then $\phi\left(z_{0}\right) \in \mathbb{C}$ with $\phi\left(z_{0}\right) \neq-1 / \sigma\left(z_{0}\right)$, and hence $M\left(z_{0}, \cdot\right)$ is a nondegenerate Mobius transformation taking $\sigma\left(z_{0}\right)$ to $\infty$. If $\sigma$ has a pole of order $m$ at $z_{0}$, then $\phi$ has a pole of the same order at $z_{0}$, so multiplying numerator and denominator by $\left(z-z_{0}\right)^{m}$, we see that $M\left(z_{0}, w\right)=c w$ for some $c \neq 0$. Hence $M$ is biholomorphic and $M(\Sigma)$ is the infinity section, so $M(D)$ is contained in $R \times \mathbb{C}$.

Now, near a generic point in $R, M(D)$ is the union of the graphs of two holomorphic functions $u_{0}$ and $u_{1}$, and the symmetric function $u_{0}+u_{1}$ is holomorphic on $R$. Hence the map

$$
N(z, w)=\left(z, w-\frac{u_{0}(z)+u_{1}(z)}{2}\right)
$$

is biholomorphic on $R \times \mathbb{P}^{1}$, and taking $h(z)=\left(u_{0}(z)-u_{1}(z)\right)^{2} / 4$, we have $N(M(D))=\left\{(z, w): w^{2}=h(z)\right\}$.

Finally, we are ready for the construction of a dominating map into the complement of a double section. 
Proof of Theorem 1.2. Let $D$ be a double section in $R \times \mathbb{P}^{1}$. By the corollary, we may assume that $D=\left\{(z, w): w^{2}=h(z)\right\}$ for some $h$ holomorphic on $R$. Let

$$
H(z, w)=\sqrt{h(z)} \frac{\exp (\sqrt{h(z)} w)+\exp (-\sqrt{h(z)} w)}{\exp (\sqrt{h(z)} w)-\exp (-\sqrt{h(z)} w)}
$$

Note that $H$ is independent of the choice of $\pm \sqrt{h(z)}$. Moreover, $H$ is simply the Mobius transformation $x \mapsto(x+1) /(x-1)$ applied to $\exp (2 \sqrt{h(z)} w)$, then multiplied by $\sqrt{h(z)}$. In particular, if $h\left(z_{0}\right) \neq 0$, then $H\left(z_{0}, \cdot\right)$ maps $\mathbb{C}$ into $\mathbb{P}^{1} \backslash$ $\{ \pm \sqrt{h(z)}\}$, and $F$ is holomorphic except possibly where $h(z)=0$.

Suppose $h\left(z_{0}\right)=0$, and without loss take $z_{0}=0$. Then $h(z)=z^{m} h_{1}(z)$ for some $m \geq 1, h_{1}(0) \neq 0$. Near 0 we have

$$
\begin{aligned}
H(z, w) & =\sqrt{h(z)} \frac{(1+\sqrt{h(z)} w)+(1-\sqrt{h(z)} w)+O\left(|z|^{(m+1) / 2}\right)}{(1+\sqrt{h(z)} w)-(1-\sqrt{h(z)} w)+O\left(|z|^{(m+1) / 2}\right)} \\
& =\frac{1+O\left(|z|^{1 / 2}\right)}{w+O\left(|z|^{(m+1) / 2}\right)} .
\end{aligned}
$$

Hence for each fixed $w \neq 0, H(\cdot, w)$ has a removable singularity at 0 , and $H(0, w)=1 / w$. So $H$ is holomorphic in each variable separately for $w \neq 0$, hence holomorphic by Hartogs' theorem as we have seen that $H$ is holomorphic for $z \neq 0$. Moreover, for each fixed $z, H$ has the form of an exponential map followed by a Mobius transformation if $D$ has two points in the fiber above $z$, and $H$ has the form of a Mobius transformation if $D$ has one point in the fiber above $z$.

2.3. Jet prescriptions. In this section we prove Theorem 1.6. So, let $D$ be a double section over $R$ and let $E$ and $P_{c}$ be as in the statement of the theorem. As before, we can interpolate on $R$, so there exists a function $\Psi$ meromorphic on $R$ such that if $c \in E$, then the Laurent expansion of $\Psi$ at $c$ agrees with $P_{c}$ to order $\operatorname{deg}\left(P_{c}\right)$. We may assume also that the graph of $\Psi$ is not contained in a component of $D$. As in the proof of Corollary 1.4, we can make a global biholomorphic change of variables so that the graph of $\Psi$ becomes the infinity section in the new coordinates. Then the requirement of agreeing with $P_{c}$ becomes the requirement of having a pole of at least some given order $n_{c}$ at each $c \in E$.

Let $p$ be a holomorphic function on $R$ with a zero of order $n_{c}$ at each $c \in E$ and no other zeros, and let $\Phi(z, w)=(z, p(z) w)$. Then $\Phi(D)$ is a double section over $R$. We may assume that $n_{c}$ is chosen large enough that if $c \in E$, then $\Phi(D)$ intersects the fiber over $c$ in the single point 0 . By Theorem 1.2, there exists a dominating map, $G$, from $R \times \mathbb{C}$ onto $\left(R \times \mathbb{P}^{1}\right) \backslash \Phi(D)$ of the form $G(z, w)=(z, L(z, w))$ as described in that theorem.

To finish the proof of the current theorem, let $F(z, w)=\Phi^{-1} G(z, w)=$ $(z, L(z, w) / p(z))$. If $c \notin E$, then $F(c, \cdot)$ is easily seen to map $\mathbb{C}$ onto $\mathbb{P}^{1} \backslash D_{c}$, 
while if $c \in E$ and $\sigma$ is holomorphic in a neighborhood of $c$, then $L(z, \sigma(z))$ is meromorphic and nonzero in a neighborhood of $c$, hence $F(z, \sigma(z))$ has a pole of order at least $n_{c}$ at $c$. Thus $F$ satisfies all the requirements.

3. The Jacobian fibration route. In this section we utilize and extend the theory of Jacobian fibrations introduced by Kodaira in [Kod] to obtain the simultaneous uniformization result of Theorem 1.5. Elementary lemmas and arguments will be given in an attempt at wider accessibility. Nevertheless, details on many of the background material can be found in [BPV], especially section V.9, and the references therein.

Our starting setup is a $\mathbb{P}^{1}$-bundle $p: P \rightarrow R$ with a distinguished double section $D$. A fiber here is just $P_{z}=\mathbb{P}^{1}$ marked with an effective divisor $D_{z}$ of degree 2. In the spirit of Kodaira, there are two simple ways to attach a nontrivial Jacobian, or more precisely a Picard variety, to this marked fiber by turning it into a singular curve. One way is to take the Jacobian of the singular curve obtained by "collapsing, to first order," the divisor $D_{z}$ into one point, resulting in a reduced and irreducible curve isomorphic to either a nodal or a cuspidal cubic curve in $\mathbb{P}^{2}$. This can be done via the linear system generated by $D_{z}+q_{1}, D_{z}+q_{2}$ and $3 q_{1}$ where $q_{1}$ and $q_{2}$ are points in $P_{z}$ chosen in general position with respect to $D_{z}$. The other way is to add to $P_{z}$, which is a smooth rational curve, another smooth rational curve intersecting $P_{z}$ at $D_{z}$ counting multiplicity. The combined object can again be realized as one of two types of plane cubic curves, but which are reducible, corresponding to the reduced total transform of the two types of irreducible plane cubic curves above blown up at their singular point. Although these methods are equivalent, and both will be introduced below, we will focus on the second one, which is easier to introduce.

We first recall that the Picard variety of a one dimensional projective scheme $Z$ is the space $\operatorname{Pic}^{0}(Z)$ of analytic line bundles with vanishing first Chern class on $Z$, where the first Chern class $c_{1}$ is defined by the connecting homomorphism $\delta$ in the long exact cohomology sequence over $Z$ of the exponential sequence (i.e., the short exact sequence $0 \rightarrow \mathbb{Z} \rightarrow \mathcal{O} \rightarrow \mathcal{O}^{*} \rightarrow 0$ ):

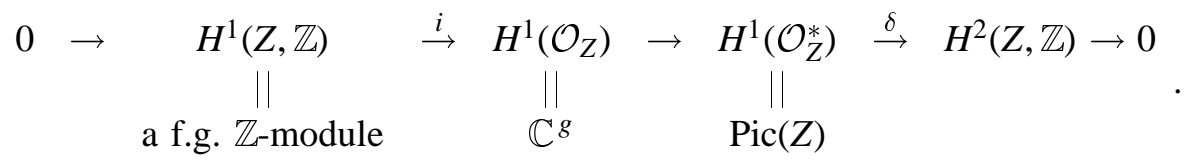

Here, $g=p_{a}(Z)$ is the arithmetic genus of $Z$ and $\operatorname{Pic}(Z)$ the space of holomorphic line bundles over $Z$. If $Z$ is reduced on the smooth part $Z_{i}^{\circ}$ of an irreducible component $Z_{i}$ of $Z_{\text {red }}$, then every line bundle $L$ on $Z$ can be written as $\mathcal{O}\left(E+E^{\prime}\right)$ for a divisor $E=\sum n_{j} s_{j}$ on $Z_{i}^{\circ}\left(n_{j} \in \mathbb{Z}, s_{j} \in Z_{i}^{\circ}\right)$ and a divisor $E^{\prime}$ on $Z \backslash Z_{i}$. Further, if $L$ is trivial on the Zariski closure of $Z \backslash Z_{i}$, then we can take $\delta(L)$ to be $\operatorname{deg} E=\sum n_{j}$. We note that $\operatorname{Pic}^{0}(Z)$ has a natural structure of a complex Lie group given by the tensor product of line bundles. Also, in the case when 
$g=1, Z_{i}^{\circ}$ can be shown to be biholomorphic to $\operatorname{Pic}^{0}(Z)$ via Serre duality and the Riemann-Roch theorem for singular curves (c.f. section V.9 of [BPV]). This fact is well understood classically for reduced plane cubic curves and the construction to see this is standard. However, for the benefit of readers who are not familiar with the projective geometry of the plane cubic curves, an exposition of this simple but beautiful, explicit classical construction will be given in the proof of the lemma to follow (see, for instance, examples 6.10.1 and 6.11.4 of [Ha]).

As a preamble, we note that all reduced plane cubic curves occur in Kodaira's table of singular fibers; see table 3 in Chapter V of [BPV]. Indeed, the classical formula for the arithmetic genus $g=(d-1)(d-2) / 2$, where $d$ is the degree of the plane curve, shows that they all have $g=1$. They are classified as Kodaira's $\mathrm{I}_{0}$, corresponding to the nonsingular elliptic curves, $\mathrm{I}_{1}$ and $\mathrm{II}$, corresponding to the plane cubic curves with a node and the ones with a cusp (i.e., rational curves with a node and ones with a cusp), $\mathrm{I}_{2}$ and III, corresponding to the reduced total transform of the blowup of the above two types (respectively) at their singular points in the plane, and IV, corresponding to the reduced total transform of the blowup of type III at the singular point. We remark that each type of fiber occuring in Kodaira's list can be regarded as a plane cubic via Riemann Roch, and we assume this in the following proof. Of the singular plane cubic curves, only those of type $\mathrm{I}_{1}$ and II are irreducible and they will serve as the starting point for the proof below.

LEMMA 3.1. Let $Z$ be a reduced plane cubic curve with a base point $\sigma \in Z_{i}^{\circ}, a$ component of the smooth locus of $Z$. Then $Z_{i}^{\circ}$ is biholomorphic to $\operatorname{Pic}^{0}(Z)$ via the map

$$
x \in Z_{i}^{\circ} \stackrel{f}{\mapsto} \mathcal{O}(x-\sigma) \in \operatorname{Pic}^{0}(Z) .
$$

Proof. We first show that $f$ is one-to-one for the case $Z$ is an irreducible cubic curve with a node (type $\mathrm{I}_{1}$ ) and the case with a cusp (type II). Assume not, so that $\mathcal{O}(x-\sigma)=\mathcal{O}\left(x^{\prime}-\sigma\right)$ where $x \neq x^{\prime}$. Then $\mathcal{O}\left(x-x^{\prime}\right)$ corresponds to the trivial line bundle over $Z$ and so $Z$ has a rational function with a simple pole at $x^{\prime}$ and a simple zero at $x$. This gives a birational morphism from $Z$ to $\mathbb{P}^{1}$. But any such morphism composed with the normalization morphism for $Z$ would give an automorphism of $\mathbb{P}^{1}$ that factors through $Z$ which is impossible since then two different points (infinitely near in the cuspidal case) of $\mathbb{P}^{1}$ would map to the same point by this automorphism.

Since the other types of reduced singular cubics can be regarded as the reduced total transforms of the above two cases via the blowing up process, the same argument as above works for them because the birational morphism from $Z_{i}$ to $\mathbb{P}^{1}$ obtained as above would factor through the corresponding irreducible cubic curves considered above. The case of the smooth cubic also follows the same argument via the birational invariance of the geometric genus for smooth curves, which is 0 for $\mathbb{P}^{1}$. Hence $f$ is one-to-one for all the cases of plane cubics. 
We next show that $f$ is onto. Again, we do this first for the two cases of $Z$ corresponding to that of an irreducible plane cubic with a node and that with a cusp. In these cases, we may write $Z_{i}^{\circ}$ simply as $Z^{\circ}$. Consider a tangent line to an inflection point $o$ on $Z^{\circ}$ (there are exactly 9 of these on any irreducible plane cubic, all ordinary), which would meet $Z$ in the divisor $3 o$. Any other divisor $H$ obtained by linear intersection would be linearly equivalent to $3 o$, and we denote this by $H \sim 3 o$. Let $L \in \operatorname{Pic}^{0}(Z)$. We may write $L \otimes \mathcal{O}(\sigma-o)$ in the form $\mathcal{O}(E)$ for a divisor $E=\sum n_{j} s_{j}$ with $n_{j} \in \mathbb{Z}$ and $s_{j} \in Z^{\circ}$ for all $j$ and $\sum n_{j}=0$. It follows that $E=\sum n_{j}\left(s_{j}-o\right)$ also. Now, given a point $s \in Z^{\circ}$, consider the line intersecting $Z$ at the divisor $o+s+t$, where $t \in Z^{\circ}$ by counting multiplicity of the intersection. Then $o+s+t \sim 3 o$, giving $s-o \sim-(t-o)$. This allows us to write $E=\sum n_{j}\left(s_{j}-o\right)$ with all $n_{j}>0$. We now show by induction on $\sum n_{j}$ that $E \sim s-o$ for some $s \in Z^{\circ}$. So suppose $\sum n_{j} \geq 2$ and let $s, s^{\prime}$ be two of the points $s_{j}$ (maybe the same) occurring in the expression for $E$. We now consider the lines intersecting $Z$ at $s+s^{\prime}+t$ and at $o+t+z$. As before, we see $t, z \in Z^{\circ}$ and $(s-o)+\left(s^{\prime}-o\right) \sim(z-o)$ which allows us to reduce $\sum n_{j}$ by 1 and consequently $E \sim s-o$ for some $s \in Z^{\circ}$ via induction. Thus $L=\mathcal{O}(E-\sigma+o)=\mathcal{O}(s-\sigma)$ for some $s \in Z^{\circ}$ and so $f$ is onto in these two cases of $Z$. The other cases of singular $Z$ now follow directly as before since they are constructed from the above cases via blowing up. Finally, the case of the smooth cubic also follows verbatim the argument above.

We have in fact exhibited the Lie group structure imposed by $\mathrm{Pic}^{0}$ on the smooth part of the cubic curves above via linear intersection. Note that the third point in a linear intersection of a cubic above depends holomorphically on the other two. It is now a formality to verify that $f$ is holomorphic. This concludes our proof.

We should point out that a common but more abstract and unified approach to show the surjectivity of $f$, without reference to which cubic, is to apply the Riemann Roch theorem and Serre duality for an embedded curve to the line sheaf $L(\sigma)$ where the dualizing sheaf of our curve $Z$ is just $\mathcal{O}_{Z}$ by the adjunction formula (see sections II.1-6 of [BPV]).

We will introduce the Jacobian fibration for our $\mathbb{P}^{1}$-bundle $p: P \rightarrow R$ endowed with the double section $D$ as the Jacobian fibration $\operatorname{Jac}(\bar{p})$ of an associated family $\bar{p}: \bar{P} \rightarrow R$ of singular plane cubic curves over $R$. The most direct way to do this is to realize $p$ as a family of conics (curves of degree two) in a $\mathbb{P}^{2}$ bundle over $R$ via the canonical embedding given by $D$ and attach to it the family of lines specified by $D$. More specifically:

PROPOSITION 3.2. With the setup as above, $\mathcal{E}=p_{*} \mathcal{O}(D)$ is a locally free sheaf of rank 3 over $R$. The natural morphism $\Phi_{D / R}: P \rightarrow \mathbb{P}(\mathcal{E})$ is an embedding with $\mathcal{O}(D)=$ $\left(\Phi_{D / R}\right)^{*} \mathcal{O}_{\mathbb{P}(\mathcal{E})}(1)$. Furthermore, $\mathcal{O}_{\mathbb{P}(\mathcal{E})}(1)$ has a section $\nu$ such that $\left(\Phi_{D / R}\right)^{*}(\nu)=D$.

Proof. Since $\mathcal{O}(D)_{z}$ is a divisor of degree 2 on $P_{z}=\mathbb{P}^{1}$, it is simply $\mathcal{O}_{\mathbb{P}^{1}}(2)$ for all $z$. So $h^{0}\left(\mathcal{O}(D)_{z}\right):=\operatorname{dim} H^{0}\left(\mathcal{O}(D)_{z}\right)=3$ is independent of $z$ and our first claim 
follows from Grauert's theorem, Theorem 12.9 of [Ha, $\S \mathrm{III}]$, as $p$ is flat. Now, the base change property of the 0th direct image map from the same theorem coupled with Nakayama's lemma shows that the natural map $p^{*} \mathcal{E} \rightarrow \mathcal{O}(D)$ is surjective as $\mathcal{O}(D)_{z}$ is generated by global sections. Hence the dual of this natural map projectivizes to a morphism $\Phi_{D / R}: P \rightarrow \mathbb{P}(\mathcal{E})$ with $\mathcal{O}(D)=\left(\Phi_{D / R}\right)^{*} \mathcal{O}_{\mathbb{P}(\mathcal{E})}(1)$. This gives an embedding on each fiber as $\mathcal{O}(D)_{z}=\mathcal{O}_{\mathbb{P}^{1}}(2)$ is very ample. Hence $\Phi_{D / R}$ is an embedding and, by definition, $D$ corresponds to a section of $\mathcal{O}_{\mathbb{P}(\mathcal{E})}(1)$.

We may now take $\bar{P}$ to be the union of $\Phi_{D / R}(P)$ and the two dimensional surface corresponding to $(\nu)$ in $\mathbb{P}(\mathcal{E})$ and take $\bar{p}$ to be the bundle projection of $\mathbb{P}(\mathcal{E})$ restricted to $\bar{P}$. By construction, $\bar{p}$ is easily verified to give a flat family of reduced schemes. (A less direct way to see this is to show that there are no embedded points in codimension two as $\bar{p}$ is flat from the general theory, such as Proposition 9.7 of $[\mathrm{Ha}, \S \mathrm{III}]$. But this is clear from the fact that all reduced plane cubic curves have arithmetic genus $g=1$, and an embedded point would increase the arithmetic genus, which would then contradict the fact that arithmetic genus stays constant in a flat family.) Note that each fiber of $\bar{p}$ as a scheme is a singular cubic curve with two irreducible components consisting of a line and a conic intersecting precisely at $\Phi_{D / R}(D)$. Note also that $p=\bar{p} \circ \Phi_{D / R}$ by construction.

The exponential sequence on $\bar{P}$ leads to the exact sequence

$$
0 \rightarrow \bar{p}_{*} \mathbb{Z} \rightarrow \bar{p}_{*} \mathcal{O}_{\bar{P}} \rightarrow \bar{p}_{*} \mathcal{O}_{\bar{P}}^{*} \rightarrow \bar{p}_{* 1} \mathbb{Z} \rightarrow \bar{p}_{* 1} \mathcal{O}_{\bar{P}}
$$

where the $\emptyset$ th and the first direct image of $\mathcal{O}_{\bar{P}}$ are locally free sheaves of rank one by Gauert's theorem as $\bar{p}$ is flat and $g$ is constant. In fact, the natural structure map $\mathcal{O}_{R} \rightarrow \bar{p}_{*} \mathcal{O}_{\bar{P}}$ generates the stalk everywhere by the base change property so that $\bar{p}_{*} \mathcal{O}_{\bar{P}}=\mathcal{O}_{R}$ forcing $\bar{p}_{*} \mathcal{O}_{\bar{P}}^{*}=\mathcal{O}_{R}^{*}$ and $\bar{p}_{*} \mathbb{Z}=\mathbb{Z}_{R}$ as both are subsheaves. It follows that the beginning of the above sequence is nothing but the exponential sequence over $R$ so that we can regard $\bar{p}_{* 1} \mathbb{Z}$ as a subsheaf of $\bar{p}_{* 1} \mathcal{O}_{\bar{p}}$. The latter, being locally free, is the sheaf of sections of a line bundle $L$ over $R$ and so includes $\bar{p}_{* 1} \mathbb{Z}$ as a subset.

To construct our Jacobian fibration, we first form the $\mathcal{O}_{R}$ module

$$
\mathcal{J a c}(\bar{p})=\bar{p}_{* 1} \mathcal{O}_{\bar{P}} / \bar{p}_{* 1} \mathbb{Z}
$$

over $R$. We would like to construct a holomorphic quotient space of $L$ by $\bar{p}_{* 1} \mathbb{Z}$ so that $\mathcal{J} a c(\bar{p})$ corresponds to the sheaf of sections of

$$
\operatorname{Jac}(\bar{p}):=L / \bar{p}_{* 1} \mathbb{Z}
$$

which would then be a holomorphic fibration of complex Lie groups with a zero section and which we will define as our Jacobian fibration. This quotient space 
is certainly possible in a neighborhood of any point where the double section $D$ consists of two distinct points since $\bar{p}_{* 1} \mathbb{Z}$ is a closed submanifold of $L$ generated over $\mathbb{Z}$ by a nonvanishing section of $L$ there. To see that $\bar{p}_{* 1} \mathbb{Z}$ is actually closed everywhere in $L$ it is necessary only to observe that in a neighborhood of any point where $D$ becomes a double point, the double valued generator over $\mathbb{Z}$ of $\bar{p}_{* 1} \mathbb{Z}$ becomes a nonvanishing meromorphic single valued generator after pulling back the fibration $p$ and hence $\bar{p}$ via the normalization of $D$, showing that the original double valued generator approaches $\infty$ at this double point. Hence it is straightforward to construct the quotient space (see [Kod], compare also [BPV, V.9] and Grothendieck [Gro], who showed that Jac exists as a scheme for any flat family) where the quotient map is a local isomorphism. This quotient is thus a complex manifold with a canonical section over $R$ coming from the zero section of $L$. In fact, we have the following proposition, which should be compared with Proposition 9.1 of [BPV, V.9].

PROPOSITION 3.3. With the setup as above, suppose that $p$ has a holomorphic section $\sigma: R \rightarrow P$ disjoint from $D$. Then there is a canonical fiber-preserving isomorphism $h$ from $\operatorname{Jac}(\bar{p})$ onto $P^{\circ}=P \backslash D$ mapping the zero-section in $\operatorname{Jac}(\bar{p})$ onto $\sigma(R)$.

Proof. The base change property shows that $\operatorname{Pic}^{0}\left(\bar{P}_{z}\right)=\operatorname{Jac}(\bar{p})_{z}$ thanks to the flatness of $\bar{p}$ and the constancy of the arithmetic genus of its fibers. Let $P^{\prime}$ be the irreducible component of $\bar{P}$ corresponding to the reduced divisor $(\nu)$ in our construction of $\bar{P}$ above. Then $P^{\circ}$ is isomorphic to $\bar{P} \backslash P^{\prime}$ under $\Phi_{D / R}$ where $P_{z}^{\circ}$ is identified with the component of the smooth part of $\bar{P}_{z}$ containing $\sigma(z)$. Consider the map $J: P^{\circ} \rightarrow \operatorname{Jac}(\bar{p})$ given on each fiber by

$$
x \in P_{z}^{\circ} \mapsto \mathcal{O}(x-\sigma(z)) \in \operatorname{Pic}^{0}\left(\bar{P}_{z}\right) .
$$

Lemma 3.1 shows that this is a bijective map that is holomorphic in the fiber direction. It is trivial to verify that $J$ maps holomorphic sections to the same locally. Hence $J$ is biholomorphic and we may set $h=J^{-1}$.

It is important to note that sections of $\operatorname{Jac}(\bar{p})$, and hence of $\mathcal{O}(L)=\bar{p}_{* 1} \mathcal{O}_{\bar{P}}$, act via addition on $P \backslash D$ as soon as $P \backslash D$ has a section by this proposition.

Theorem 1.5 is a corollary of this proposition. Moreover, using this proposition, we now give a second proof of Theorem 1.3 following the proof of Lemma 3.8 in [BL] with some needed clarification. Theorem 1.2 then clearly follows by a final application of the proposition.

Second proof of Theorem 1.3. With the setup as above, the hypotheses of theorem 1.3 allow us to assume that $R$ is noncompact and $P=R \times \mathbb{P}^{1}$ with $p$ being the projection to the first factor. We will exploit the fact that $R$, being Stein, contains an one-dimensional simplicial complex $K$ which is a strong deformation retract of $R$ (see [GR, $\S \mathrm{V} .4 .5]$ ). 
We first assume that $D$ has no double point at any fibre, which is equivalent to assuming that $\Gamma=\bar{p}_{* 1} \mathcal{O}_{\bar{P}}$ is a locally constant sheaf, or equivalently, a local system. Then the pair $\left(K,\left.\Gamma\right|_{K}\right)$ is a deformation retract of $(R, \Gamma)$. Hence $H^{2}(R, \Gamma)=$ $H^{2}(K, \Gamma)$, which is zero as it is isomorphic to the simplicial sheaf cohomology of a simplicial complex (since $\Gamma$ restricted to any simplex is a constant sheaf and hence has trivial cohomology, leading to the degeneration at $E_{1}$ of the double complex comparing simplicial and ordinary sheaf cohomology) which has dimension less than two. Since the cohomology groups of $L$ are trivial as $R$ is Stein, the long exact sequence of the short exact sequence

$$
0 \rightarrow \Gamma \rightarrow L \rightarrow \operatorname{Jac}(\bar{p}) \rightarrow 0
$$

(as sheaves) shows that $H^{1}(R, \operatorname{Jac}(\bar{p}))=H^{2}(R, \Gamma)$. Hence $H^{1}(R, \operatorname{Jac}(\bar{p}))=0$.

Now, every point on $R$ admits a neighborhood with a section of $p$ avoiding $D$. We choose a locally finite good covering of $R$ by distinct open sets $U_{1}, U_{2}, \ldots$, with sections $\tau_{1}, \tau_{2}, \ldots$ of $p$ over $U_{1}, U_{2}, \ldots$, respectively. By Proposition 3.3, there is a unique section $\tau_{i j}^{\prime} \in H^{0}\left(U_{i j}, \operatorname{Jac}(\bar{p})\right)$ such that $\tau_{i}+\tau_{i j}^{\prime}=\tau_{j}$ on $U_{i} \cap U_{j}$ for all $i, j$. It is clear that $\left\{\tau_{i j}^{\prime}\right\}$ satisfies the cocycle condition. Hence, so does $\left\{-\tau_{i j}^{\prime}\right\}$, which therefore defines an element in $H^{1}\left(\left\{U_{i}\right\}, \operatorname{Jac}(\bar{p})\right)=H^{1}(R, \operatorname{Jac}(\bar{p}))$ (where the last equality follows from Leray's theorem [GR, $\S \mathrm{B} .3 .3]$ ). Hence $\left\{-\tau_{i j}^{\prime}\right\}$ is a coboundary and one can find holomorphic sections $\tau_{i}^{\prime} \in H^{0}\left(U_{i}, \operatorname{Jac}(\bar{p})\right)$ such that $\tau_{i}^{\prime}-\tau_{i j}^{\prime}=\tau_{j}^{\prime}$. Then $\tau_{i}+\tau_{i}^{\prime}=\tau_{j}+\tau_{j}^{\prime}$ on $U_{i} \cap U_{j}$ for all $i, j$. This gives rise to a global section of $p$ avoiding $D$.

In the general case, the problem again reduces to the vanishing of $H^{2}(R, \Gamma)$, which allows us to lift the $\tau_{i j}^{\prime} s$ as above to sections of $L$ (by assuming without loss that $D$ consists of two disjoint components over $U_{i} \cap U_{j}$ ) that satisfy the cocycle condition and thus giving an element of $H^{1}\left(\left\{U_{i}\right\}, L\right)$, which is zero as $R$ is Stein. This can be done via an elementary combinatorial argument using $K$ (see [Lu]), but we offer here an alternative to $H^{2}(R, \Gamma)$ :

Let $E_{0}$ be the set of points above which $D$ is a double point. Then $E_{0}$ is a closed discrete subset of $R$. Note that the closure $A$ in $R$ of a component of $R \backslash K$, being contractible modulo its boundary $\partial A$ in $R$, is homeomorphic to either the complement of $\mathbb{D}$ with some identifications on the boundary circle or to the upper half plane in $\mathbb{C}$. It follows that any closed discrete subset of $A$ is contained in $\partial A$ union a simple (without self-intersection) path in $A \backslash \partial A$ connecting $\partial A$ with the complement of every compact subset of $A$ (by using, for example, the discrete set of circles in $\mathbb{C}$ centered at the origin which together contain the discrete subset corresponding to $E_{0} \cap A$ ). Hence we may define a one-dimensional simplicial complex $J$ containing $K$ and $E_{0}$ as the union of $K$ with a set of such paths, one path for each component of $R \backslash K$. Then $\Gamma$ is locally constant on $R^{\prime}:=R \backslash J$ and each component of $U \backslash J$ is contractible for every deformation retract neighborhood $U$ of $J$ in $R$. We take such a neighborhood $U$ small enough so that it has a covering by distinct open subsets $U_{1}, U_{2}, \ldots$ of $U$ without triple intersections and with 
sections $t_{1}, t_{2}, \ldots$ of $p$ over $U_{1}, U_{2}, \ldots$, respectively, all avoiding $D$. We may further stipulate that $D$ consists of two disjoint components over each double intersection $U_{i j}, i \neq j$. Then $\Gamma$ is locally constant on $U_{i j}$, hence constant, giving $H^{1}\left(U_{i j}, \Gamma\right)=0$. It follows that the section $t_{i j}^{\prime}$ of $\operatorname{Jac}(\bar{p})$ over $U_{i j}$ constructed via Proposition 3.3 to satisfy $t_{i}+t_{i j}^{\prime}=t_{j}$ lifts to a section $\hat{t}_{i j}$ of $L$ over $U_{i j}$ by the long exact sequence of (3.1). As $\left\{\hat{t}_{i j}\right\}$ trivially satisfy the cocycle condition and as $H^{1}(U, L)=0$, there exist sections $\hat{t}_{i} \in H^{0}\left(U_{i}, L\right)$ such that $\hat{t}_{i}-\hat{t}_{i j}=\hat{t}_{j}$. Then $\hat{t}_{i}+t_{i}=\hat{t}_{j}+t_{j}$ on $U_{i j}$ for all $i \neq j$. This gives rise to a global section of $p$ avoiding $D$ over $U$.

Finally, $R$ is covered by the open sets $R^{\prime}$ and $U$, both of which admit sections of $p$ as shown above, and $R^{\prime} \cap U$ consists of contractible sets by construction, hence the same argument as in the last paragraph gives a global section of $p$ avoiding $D$ as required.

We note that this proof is very similar to the approach of the Oka-Grauert principle for principal (and associated) bundles over Stein spaces (see [GR] for example). However, the current proof applies to a fibration of Lie groups which is not necessarily smooth.

4. Fibered surfaces. In this section, we will give conditions under which a nonhyperbolically fibered complex surface is dominable by $\mathbb{C}^{2}$ by proving Theorem 1.7.

Recall that a nonhyperbolically fibered surface $X$ is given by a holomorphic map $f$ from $X$ onto a complex curve $C$ whose generic fiber is nonhyperbolic such that $f$ extends to a proper map from a surface $Y$ to $C$, where $Y$ contains $X$ as a Zariski open subset. We will also use $f$ to denote the extended map by abuse.

Recall also that each fiber $X_{S}$ is an effective divisor on $X$ as the pull back of a Q-divisor in $C$. We write $X_{s}=\sum n_{i} C_{i}$ where $C_{i}$ is the $i$ th irreducible component of $\left(X_{s}\right)_{\text {red }}$ and $n_{i}-1$ is the generic vanishing order of $d f$ on $C_{i}$. The positive integer coefficient $n_{i}$ is called the multiplicity of the $i$ th component. The multiplicity of a fiber $X_{s}=\sum n_{i} C_{i}$ is defined as the greatest common divisor $n_{s}$ of $\left\{n_{i}\right\}$. A fiber $X_{s}$ with $n_{s}>1$ is called a multiple fiber.

Vital to our problem is the minimum multiplicity in the fiber $X_{S}$ defined by $m_{s}=\min \left\{n_{i}\right\}$. As we will soon see, this gives the correct orbifold structure on $C$ via the $\mathbb{Q}$-divisor $A=\sum_{s}\left(1-1 / m_{s}\right) s$ in $C$ for hyperbolicity questions. Note that $X_{s}$ is a Zariski open subset of $Y_{s}$ and we will assume for clarity that the map $f$ in a neighborhood of each point $y \in Y_{s}$ is given by an equation of the form

$$
x_{1}^{n_{i_{1}}} x_{2}^{n_{i_{2}}}=t
$$

for a local coordinate system $\left(x_{1}, x_{2}\right)$ centered at $y$ and a local coordinate $t$ centered at $s$. We can always do this by further blowing up $Y$ if necessary (see Theorem V.3.9 in [Ha]). We will first prove that (c) implies (b) in Theorem 1.7. 


\subsection{Ahlfors' lemma for generalized orbifold maps.}

Definition 4.1. Let $(C, A)$ be a one dimensional complex orbifold, where the $\mathbb{Q}$-divisor $A=\sum_{s}\left(1-1 / m_{s}\right) s$ on the complex curve $C$ specifies the orbifold structure as above and where the positive integer $m_{s}$ is identically 1 except on a discrete subset of $C$. An orbifold metric on $(C, A)$ is given by a $(1,1)$-form $\rho$ on $C \backslash A_{\text {red }}$ which, in a coordinate neighborhood $U(s)$ of each $s \in C$, has the form

$$
|t|^{-2\left(1-\frac{1}{m_{s}}\right)} \mu
$$

for a smooth positive $(1,1)$-form $\mu$ on $U$ with the coordinate $t$ centered at $s$.

A holomorphic map $f$ from a complex manifold $X$ to $C$ is called a Z-orbifold map to $(C, A)$ if $f^{*} \rho$ extends to a continuous $(1,1)$-form over its singularities (if it has any) on $X$ for some (and hence any) orbifold metric $\rho$ on $(C, A)$.

A example of a Z-orbifold map is given by the fibering map $f$ of a fibered surface $X$ over a curve $C$ with the minimum multiplicity in the fibers defining the orbifold structure on $C$. One can deduce this directly from the local coordinate picture given by (4.1). Composing $f$ with any holomorphic map gives of course again a Z-orbifold map. If $(C, A)$ is hyperbolic, meaning that it is an orbifold quotient of the unit disk $\mathbb{D}$ by automorphisms of $\mathbb{D}$, then it inherits a canonical orbifold metric from the Poincaré metric $\hat{\rho}$ on $\mathbb{D}$, which is invariant under automorphisms of $\mathbb{D}$. We will call this orbifold metric the orbifold Poincaré metric and denote it by $\rho$. It is determined as an orbifold metric on $(C, A)$ by the equation

$$
d d^{c} \log \rho=\rho
$$

just as the Poincare metric on $\mathbb{D}$. With this in mind, the proof of the following version of Ahlfors' lemma follows verbatim that of the usual one and is left to the reader.

THEOREM 4.2. Denote the Poincaré metric on $\mathbb{D}$ by $\hat{\rho}$ and the orbifold Poincaré metric on a hyperbolic one-dimensional complex orbifold $(C, A)$ by $\rho$. Then any $Z$-orbifold map $h$ from $\mathbb{D}$ to $(C, A)$ satisfies the distance decreasing property in the form

$$
h^{*} \rho \leq \hat{\rho}
$$

with equality at any point in $C \backslash A$ characterizing $h$ to be the orbifold quotient map from $\mathbb{D}$.

By applying this to larger and larger disks in $\mathbb{C}$, we see that any Z-orbifold map from $\mathbb{C}$ to a hyperbolic $(C, A)$ must be constant. From the classical uniformization theorem for orbifold curves, $(C, A)$ is not hyperbolic (meaning that it is not uniformized by the disk) precisely when $C$ is quasiprojective and $\chi(C, A) \geq$ 
0 (see [BL], which explains this well-known fact from [FK, IV 9.12]). So if the latter condition does not hold, then any holomorphic image of $\mathbb{C}$ in a surface $X$ fibered over the orbifold $(C, A)$ must lie in a fiber. This shows that condition (c) of Theorem 1.7 implies condition (b).

4.2. Proof of Theorem 1.7. It is easy to deduce (c) from (a), that if there is a dominating map $F: \mathbb{C}^{2} \rightarrow X$, then there is also a holomorphic image of $\mathbb{C}$ which is Zariski dense: First we may assume that the Jacobian of $F$ is nonzero at the origin. Defining $g: \mathbb{C} \rightarrow \mathbb{C}^{2}$ by $h(z)=\left(\sin (2 \pi z), \sin \left(2 \pi z^{2}\right)\right)$, we see that $g(n)=(0,0)$ with corresponding tangent direction $(2 \pi, 4 \pi n)$ for each $n \in \mathbb{Z}$. Taking $F \circ g$, we obtain a holomorphic image of $\mathbb{C}$ with an infinite number of tangent directions at one point, which implies that the image is Zariski dense.

To complete the proof, we need to deduce (a), the existence of a dominating map from $\mathbb{C}^{2}$, from condition (b), the nonhyperbolicity of $(C, A)$. The latter implies, by the uniformization theorem, that $(C, A)$ is the orbifold quotient of $\mathbb{C}$ or $\mathbb{P}^{1}$ unless $C=\mathbb{P}^{1}$ with $\#\left\{A_{\text {red }}\right\} \leq 2$. In the "unless" case, by removing up to two fibers in $X$, we may assume that $C=\mathbb{C}^{*}$ with $A=\emptyset$ to construct our dominating map. Also, in the case $(C, A)$ is the orbifold quotient of $\mathbb{P}^{1}$, we may replace $(C, A)$ by $\left(\mathbb{P}^{1}, \emptyset\right)$ without loss and then delete a point to form $(\mathbb{C}, \emptyset)$. Hence in all the nonhyperbolic cases, we may assume that $(C, A)$ is uniformized by $\mathbb{C}$, which we assume henceforth.

Let $r: \mathbb{C} \rightarrow(C, A)$ be the uniformization map where the orbifold structure $A$ is defined by the minimum multiplicity on the fibers of $f: X \rightarrow C$. Let $\tilde{Y}$ be the minimal resolution of singularities of the normalization of the fiber product of $Y$ with $\mathbb{C}$ over $C$. $\tilde{Y}$ naturally fibers over $\mathbb{C}$ as the pullback fibration of $Y$ over $C$ via $r$. Take $\tilde{X}$ to be the inverse image of $X$ in $\tilde{Y}$. Then $\tilde{X}$ is a Zariski open subset of $\tilde{Y}$ with an irreducible component of multiplicity one at every fiber by construction. We will denote the union of all such irreducible components of $\tilde{X}$ minus the singular points of the fibers of $\tilde{X}$ by $\tilde{X}^{\circ}$, which is a Zariski open subset of $\tilde{X}$. It surjects to $\mathbb{C}$ under the fibration map. We get the following diagram of maps:

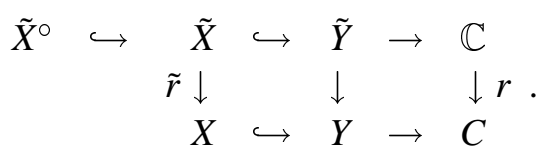

By repeatedly blowing down the $(-1)$-curves in the fibers of $\tilde{Y}$, we obtain a relatively minimal fibration $\tilde{Y}_{\text {min }}$ over $\mathbb{C}$ (a fibration without such curves on its fibers). Moreover, Proposition III.8.4 in [BPV] (or rather its proof plus the fact that the arithmetic genus of a multiple $\mathbb{P}^{1}$ fiber must be negative, which is disallowed, by II.11(b) of [BPV]) tells us that $\tilde{Y}_{\min }$ is unique unless all of its fibers over $\mathbb{C}$ are $\mathbb{P}^{1}$, which implies that $\tilde{Y}_{\text {min }}$ is a trivial $\mathbb{P}^{1}$ bundle by the Grauert-Fischer theorem and hence also unique. Since the generic fiber $X_{c}$ of $X$ is nonhyperbolic, $X_{c}$ is either $\mathbb{P}^{1}$ with at most two punctures or it is an elliptic 
curve. In the former case $\tilde{Y}_{\min }$ is a trivial $\mathbb{P}^{1}$ bundle over $\mathbb{C}$ while in the latter $\tilde{Y}_{\text {min }}$ is a relatively minimal elliptic fibration. We will call these cases case (1) and case (2), respectively, and consider them separately below.

The key is to note that, by construction, points of $\tilde{X}^{\circ}$ are infinitely near points on $\tilde{Y}_{\text {min }}$ (including $\emptyset$ th order) representing jets of local sections of $\tilde{Y}_{\min }$ over $\mathbb{C}$. Thus, if we let $\tilde{Y}_{\min }^{\circ}$ be the union of all irreducible components of multiplicity one of fibers of $\tilde{Y}_{\min }$ minus the singular points of the fibers of $\tilde{Y}_{\min }$, then $\tilde{Y}_{\min }^{\circ}$ surjects to $\mathbb{C}$. In particular, $\tilde{Y}_{\min }^{\circ}$ does not have any multiple fiber and contains the image of $\tilde{X}^{\circ}$. We have the following diagram:

$$
\tilde{p}: \tilde{X} \stackrel{\iota}{\hookrightarrow} \tilde{Y} \stackrel{j}{\rightarrow} \tilde{Y}_{\min } \stackrel{\pi}{\rightarrow} \mathbb{C} .
$$

To conclude our proof, it is sufficient to construct a dominating holomorphic map from $\mathbb{C}^{2}$ to $\tilde{X}$ since a dominating holomorphic map to $X$ can then be obtained by composing with the natural surjective holomorphic map $\tilde{r}$ from $\tilde{X}$ to $X$.

Case (1). The generic fiber $X_{c}$ is $\mathbb{P}^{1}$ with at most two punctures: In the case of exactly two punctures, $\tilde{X}$ is isomorphic via $j \circ \iota$ to the complement of a double section $D$ in the $\mathbb{P}^{1}$ bundle $\tilde{Y}_{\text {min }}$ over the complement of a discrete set $E$ in $\mathbb{C}$. Since $\tilde{p}$ maps $\tilde{X}^{\circ}$ onto $\mathbb{C}$, we may choose a point in $\tilde{X}_{s}^{\circ}$ for each $s \in E$, thereby defining a jet prescription on $\tilde{Y}_{\min }$ over $E$. Applying Theorem 1.6 then gives us a holomorphic map $F$ from $\mathbb{C}^{2}$ to $\tilde{Y}_{\text {min }}$ that surjects to the $\tilde{Y}_{\text {min }} \backslash D$ above $\mathbb{C} \backslash E$. Moreover, $F$ is fiberwise (meaning $\pi \circ F=p_{1}$, the projection to the first factor of $\mathbb{C}^{2}$ ) and maps sections of $p_{1}$ to sections of $\pi$ satisfying the jet prescription on $E$ given above. Hence $F$ lifts to a dominating holomorphic map to $\tilde{X}$ which then gives a dominating map to $X$ by composition.

The case of no puncture can be reduced to that of a puncture by removing a section from the trivial $\mathbb{P}^{1}$ bundle $\tilde{Y}_{\text {min }}$. Hence it remains only to consider the case of exactly one puncture to establish Case (1). This puncture can be regarded as the infinity section $D_{\infty}$ in the trivial $\mathbb{P}^{1}$ bundle $\tilde{Y}_{\text {min }}$, whose complement is a trivial line bundle $L$ over $\mathbb{C}$ as $\mathbb{C}$ is Stein. Again, over the complement of a discrete set $E$ in $\mathbb{C}, \tilde{X}$ is isomorphic via $j \circ \iota$ to this line bundle.

We now choose a point in $\tilde{X}_{s}^{\circ}$ for each $s \in E$, thus defining a jet prescription over $E$. Using a trivialization of $\tilde{Y}_{\min }$, this jet prescription corresponds to prescribing a Laurent polynomial at each point of $E$, and the basic interpolation theorems as before allow us to find a meromorphic function $q(z)$ on $\mathbb{C}$ satisfying this jet prescription on $E$. Let $p(z)$ be an entire function vanishing to an order greater than that of the jet prescription at each point of $E$ and nonzero outside $E$. If $w$ is the vertical coordinate of $\mathbb{C}^{2}$, then the map

$$
(z, w) \mapsto(z, p(z) w+q(z))
$$

gives a holomorphic dominating map $F$ from $\mathbb{C}^{2}$ to $\tilde{Y}_{\text {min }}=\mathbb{C} \times \mathbb{P}^{1}$ which com- 
mutes with the projection to the first factor (i.e., $F$ is a fiberwise map). It certainly maps onto $L \hookrightarrow \tilde{Y}_{\text {min }}$ outside $E$ and satisfies the jet prescriptions over $E$ for the image of all sections in $\mathbb{C}^{2}$. Hence $F$ lifts to a dominating holomorphic map to $\tilde{X}$ as required.

Case (2). The generic fiber $X_{c}$ is an elliptic curve. Then, as before, $\tilde{X}^{\circ}$ is isomorphic to $\tilde{Y}_{\min }^{\circ}$ via $j \circ \iota$ above the complement of a discrete set $E$ in $\mathbb{C}$, above which $\tilde{Y}_{\min }^{\circ}$ is in particular a smooth elliptic fibration. Choosing a point in $\tilde{X}^{\circ}$ above each point in $E$ gives a jet prescription of local sections through a component of $\tilde{Y}_{\min }^{\circ}$ above each point in $E$. If we remove all the other components of $\tilde{Y}_{\text {min }}^{\circ}$, then we will show that what remains is isomorphic to a Jacobian fibration, which is a holomorphic quotient of $\mathbb{C}^{2}$ where the quotient map is fiberwise and is a local isomorphism. Assuming we have shown this for the moment, then our jet prescription in $\tilde{Y}_{\min }^{\circ}$ above a point $s \in E$ gives rise to possibly infinitely many jet prescriptions in $\mathbb{C}^{2}$ above $s$. Choosing one such jet prescription for each $s \in E$ and arguing as in the second part of Case (1) above gives us a dominating map from $\mathbb{C}^{2}$ to itself satisfying this jet prescription on $\mathbb{C}^{2}$. Hence, composing this dominating map with the quotient map above gives us a dominating map to $\tilde{Y}_{\min }^{\circ}$ satisfying the jet prescription given there. This map would then lift to a dominating map to $\tilde{X}^{\circ}$, establishing our Theorem 1.7.

So it remains to establish the isomorphism to a Jacobian fibration as mentioned above. We will do this in complete analogy with the proof of Theorem 1.5 in Section 3. Fortunately, Kodaira in [Kod] has worked out the analogue of our Proposition 3.3 which we quote as follows (see also [BPV, §V.9]).

Proposition 4.3. Let $f: X \rightarrow C$ be a relatively minimal elliptic fibration over a curve $C$ with a holomorphic section $\sigma: C \rightarrow X$. Let $X_{\sigma}^{\prime}$ consist of all irreducible components of fibers $X_{s}$ not meeting $\sigma(C)$, and let $X^{\sigma}=X \backslash X_{\sigma}^{\prime}$. Then there is a canonical fiber-preserving isomorphism $h$ from $\operatorname{Jac}(f)$ onto $X^{\sigma}$ mapping the zerosection in $\operatorname{Jac}(f)$ onto $\sigma(C)$.

Since $\operatorname{Jac}(f)$ is a holomorphic fiberwise quotient of the trivial line bundle $L:=f_{* 1} \mathcal{O}_{X}$ over $\mathbb{C}$ (as in Section 3, see also [BL]), the proposition implies that as soon as $f$ has a section, sections of this line bundle act on $X$ by translation in the fiber direction via addition.

This proposition allows us to finish the proof by constructing a section $\sigma$ of $\tilde{Y}_{\min }^{\circ}$ intersecting the component corresponding to our jet prescription for each $s \in E$, since deleting the other components from $\tilde{Y}_{\min }^{\circ}$ gives us exactly $\tilde{Y}_{\min }^{\sigma}$ by construction. This section $\sigma$ exists by the following lemma. Its proof is verbatim that at the end of Section 3, where we emulated Oka's original approach [Oka] to the Oka-Grauert principle for bundle of Lie groups, and is fitting as a conclusion to this section.

Lemma 4.4. Given a relatively minimal elliptic fibration $f: X \rightarrow C$ without multiple fibers, assume $C$ is noncompact. Then $f$ has a holomorphic section. Fur- 
thermore, if we choose an irreducible component with multiplicity one in each fiber and let $X^{\circ}$ be their union minus the singular points of the fibers, then the above holomorphic section can be chosen to have image in $X^{\circ}$.

Proof. From Kodaira's table of nonmultiple singular fibers ([Kod] or [BPV, table 3, p. 150]), we see that every fiber which is not multiple in a relatively minimal elliptic fibration has a component of multiplicity one. We choose one such for each fiber of $f$ to construct $X^{\circ}$ as in the lemma. Then, every point on $C$ admits a neighborhood with a section in $X^{\circ}$. The rest of the proof is now verbatim that given at the end of Section 3 replacing Proposition 3.3 by Proposition 4.3.

5. Further remarks. Our main theorems on dominating maps allow us to show, for example, that blowing up $\mathbb{P}^{2}$ at $x$ and taking the complement of the strict transform of the normal crossing quartic consisting of two lines intersecting at $x$ and a conic gives us an algebraic surface that is dominated holomorphically by $\mathbb{C}^{2}$. This despite the fact that the complement of the same quartic in $\mathbb{P}^{2}$ is not so dominable, as shown in [CG] using a well-known lemma of Kodaira [K].

As for Theorem 1.3 on the existence of a section, we first note that it and hence also Theorem 1.2 are false for a compact base $R$ since the double section $D$ can be ample. It is not difficult by our method to characterize those double sections on a compact ruled surface which admit sections disjoint from it. The same characterization when $D$ is replaced by a section is classical and can be found, for example, in [Ha, $\S \mathrm{V} .2]$.

We can also consider the possibility of other generalizations of Theorem 1.3, especially various versions of the " $n$-section" problem. However, the following problem already has a negative answer for $n=3$.

Question 5.1. Let $D$ be an $n$-section of a $\mathbb{P}^{1}$ bundle over a noncompact Riemann surface. That is, $D$ is a reduced divisor intersecting each fiber exactly $n$ times counting multiplicity. Does there exist a section of the bundle avoiding $D$ ?

We have already shown that the answer is yes for $n=2$. To see that the answer is no for $n=3$, we consider the following example:

Let $L_{0}(z)=0, L_{1}(z)=z$, and let $T$ be the triple section in $\mathbb{C} \times \mathbb{P}^{1}$ given by the union of the infinity section and the graphs of $L_{0}$ and $L_{1}\left(L_{0}\right.$ and $L_{1}$ could be replaced by any two lines meeting at the origin). Suppose $\sigma$ is an entire function whose graph avoids $T$. We will exclude the fiber over $0 \in \mathbb{C}$ and pull back with the covering map to get a contradiction.

Let $\Phi(u, v)=\left(e^{u}, v\right)$, and let $T^{*}$ be $\Phi^{-1}(T)$. Then $T^{*}$ is the union of the infinity section and the graphs of $L_{0}^{*}$ and $L_{1}^{*}$, where $L_{0}^{*}(u)=0$ and $L_{1}^{*}(u)=e^{u}$. Also, $\Phi^{-1}$ of the section $\sigma$ is the graph of $\sigma^{*}(u)=\sigma\left(e^{u}\right)$, and this graph is contained in the complement of $T^{*}$. 
Finally, let $\Psi(u, v)=\left(u, v / e^{u}\right)$. Then $\Psi\left(T^{*}\right)$ is $\mathbb{C} \times\{0,1, \infty\}$, and hence $\Psi$ applied to the graph of $\sigma^{*}$ is the graph of an entire function $\sigma^{* *}$ which never attains the values 0 or 1 and which hence must be some constant $c \neq 0,1$. But also $\sigma^{* *}(u)=\sigma^{*}(u) / e^{u}=\sigma\left(e^{u}\right) / e^{u}$.

Thus $\sigma\left(e^{u}\right)=c e^{u}$, or equivalently $\sigma(z)=c z$. But then $\sigma(0)=0$, which contradicts the fact that the graph of $\sigma$ avoids $T$.

Note that this is an example over a nonhyperbolic base. The same question for a hyperbolic base curve remains an important open problem.

As for nonhyperbolically fibered surfaces, it is clear that dominability by $\mathbb{C}^{2}$ is a bimeromorphic invariant. It follows that the hyperbolicity of the orbifold base curve from the minimal multiplicities of components of the fibration is also invariant, as is the property of having a Zariski dense holomorphic image of $\mathbb{C}$ by Theorem 1.7. However, it is still a problem to find the connection of this invariant to other natural bimeromorphic invariants of the surface, especially to tensorial invariants, as one would like to find a differential geometric connection.

In this connection, $[\mathrm{BL}]$ gives a fundamental group characterization of dominability for projective algebraic surfaces modulo certain nonelliptic K3 surfaces, which we quote below.

THEOREM 5.2. A projective surface $X$ not birationally equivalent to a $K 3$ surface is dominable by $\mathbb{C}^{2}$ if and only if it has Kodaira dimension less than two and its fundamental group is a finite extension of an abelian group (of even rank four or less). If $\kappa(X)=-\infty$, then the fundamental group condition can be replaced by the simpler condition of nonexistence of more than one linearly independent holomorphic one-form. If $\kappa(X)=0$ and $X$ is not birationally equivalent to a K3 surface, then $X$ is dominable by $\mathbb{C}^{2}$. If $X$ is birationally equivalent to an elliptic $K 3$ surface or to a Kummer K3 surface, then $X$ is dominable by $\mathbb{C}^{2}$.

The proof of this characterization depends on the fact that in the case of a properly fibered surface, every nonmultiple fiber has an irreducible component of multiplicity one. This is no longer true when the fibers of a fibered surface are allowed to be noncompact. In fact, the following is a simple example of a fibered, simply connected, quasiprojective surface that is generically ruled (a $\mathbb{P}^{1}$ bundle outside some finite subset), but is not dominable by $\mathbb{C}^{2}$.

Consider the fibration $\mathbb{C} \times \mathbb{P}^{1} \rightarrow \mathbb{C}$ obtained by projecting to the first factor. Note that the base curve $C$ is quasiprojective. Consider two points $a=(0,2)$, $b=(0,3)$ in the fiber at 0 and the following operation on this surface. Take two consecutive blowups at $a$ (first blow up at $a$ and then blow up at the singular point of the resulting fiber) and three such blowups at $b$ to obtain an irreducible component of multiplicity 2 above $a$ and one of multiplicity 3 above $b$ for the resulting fiber. Delete all other components of this fiber from the blown-up surface and call the resulting open surface $Y^{*}$. 
It is easy to see that the resulting surface is simply connected: First note that any loop can be homotoped to the complement of the fiber (since the fiber is real codimension two in the surface) and so corresponds to a multiple of the generator $\alpha$ of $\pi_{1}(\mathbb{C} \backslash\{0\})$. Take a simple loop $S$ outside the fiber corresponding to $\alpha$. Compose $S$, with a base point fixed, with two loops outside the fiber: one corresponds to $-3 \alpha$ in a disk through $b$ transversal to the fiber and the other corresponds to $2 \alpha$ in a disk through $a$ transversal to the fiber. Since these two loops are contractible in $Y^{*}$, the composition is homotopic to $S$ but corresponds to the zero multiple of $\alpha$ and hence is homotopically trivial as the generic fiber is simply connected. So, $Y^{*}$ is simply connected. Note that the fiber $Y_{0}^{*}$ is not a multiple fiber as the multiplicities of its components are relatively prime.

Now we do exactly the same operation to two additional fibers giving a surface $Y^{* *}$ which is simply connected by the same argument as above. However, the orbifold Euler characteristic for the base orbifold curve using the minimal multiplicity of the components of the fibers of $Y^{* *}$ is $1-3(1-1 / 2)=-1 / 2<0$, showing that the orbifold curve is hyperbolic. Hence any holomorphic image of $\mathbb{C}$ in $Y^{* *}$ must lie in a fiber as a consequence of our Ahlfors' lemma, Theorem 4.2.

DEPARTMENT OF MATHEMATICS, CORNELl University, IthaCA, NY 14853

DEPARTMENT OF Mathematics, UniVERSity of WATERLOO, WATERLOO, ONTARIO, N2L 3G1, CANADA

REFERENCES

[BPV] W. Barth, C. Peters, and A. Van de Ven, Compact Complex Surfaces, Springer-Verlag, Berlin, 1984.

[BL] G. Buzzard and S. Lu, Algebraic surfaces holomorphically dominable by $\mathbb{C}^{2}$, Invent. Math. (to appear).

[CG] J. Carlson and P. Griffiths, A defect relation for equidimensional holomorphic mappings between algebraic varieties, Ann. of Math. 95 (1972), 557-584.

[C] E. Chirka, Complex Analytic Sets, Kluwer Academic Publishers, Dordrecht, 1989.

[FK] H. M. Farkas and I. Kra, Riemann Surfaces, Graduate Texts in Math, vol. 71, Springer-Verlag, New York, 1980.

[F] O. Forster, Lectures on Riemann Surfaces, Graduate Texts in Mathematics, vol. 81, Springer-Verlag, New York, 1981.

[GR] H. Grauert and R. Remmert, Theory of Stein Spaces, Grundlehren Math. Wiss., vol. 236, SpringerVerlag, Berlin, 1979.

[Gro] A. Grothendieck, Fondements de la Géométrie Algébrique, Secrétariat Math., Paris, 1962.

[Ha] R. Hartshorne, Algebraic Geometry, Graduate Texts in Math, vol. 52, Springer-Verlag, New York, 1977.

[Kod] K. Kodaira, On compact complex analytic surfaces I, Ann. of Math. 71 (1960), 111-152; II, Ann. of Math. 77 (1963), 563-626; III, Ann. of Math. 78 (1963), 1-40.

[Kod1] _ On the structure of compact complex analytic surfaces, Lecture notes prepared in connection with the AMS Summer Institute on Algebraic Geometry held at the Whitney Estate, Woods Hole, MA, July, 1964. 
[Kod2] , On the structure of compact complex analytic surfaces I, Amer. J. Math. 86 (1964), 751-798.

[K] Pluricanonical systems on algebraic surfaces of general type, J. Math. Soc. Japan 20 (1968), 170-192.

[Lu] S. S.-Y. Lu, Multiply marked Riemann surface and the Kobayashi pseudometric on algebraic manifolds, Fields Institute preprint, 2000.

[Oka] K. Oka, Sur les fonctions analytiques de plusieurs variables. III. Deuxième probléme de Cousin, $J$. Sci. Hiroshima Univ. Ser. A 9 (1939), 7-19. 